\title{
Le numérique : une bibliothèque universelle pour la création scientifique?
}

Jean-Pierre Elloy et Morgan Magnin

\section{OpenEdition}

1 Journals

Édition électronique

URL : https://journals.openedition.org/rbnu/1642

DOI : $10.4000 /$ rbnu. 1642

ISSN : 2679-6104

Éditeur

Bibliothèque nationale et universitaire de Strasbourg

Édition imprimée

Date de publication : 1 novembre 2014

Pagination : 68-75

ISBN : 9782859230548

ISSN : 2109-2761

\section{Référence électronique}

Jean-Pierre Elloy et Morgan Magnin, « Le numérique : une bibliothèque universelle pour la création scientifique? ", La Revue de la BNU [En ligne], 10 | 2014, mis en ligne le 01 novembre 2014, consulté le 19 mai 2021. URL : http://journals.openedition.org/rbnu/1642 ; DOI : https://doi.org/10.4000/rbnu. 1642

\section{(c) (1) ()}

La Revue de la BNU est mise à disposition selon les termes de la Licence Creative Commons Attribution - Pas d'Utilisation Commerciale - Partage dans les Mêmes Conditions 4.0 International. 


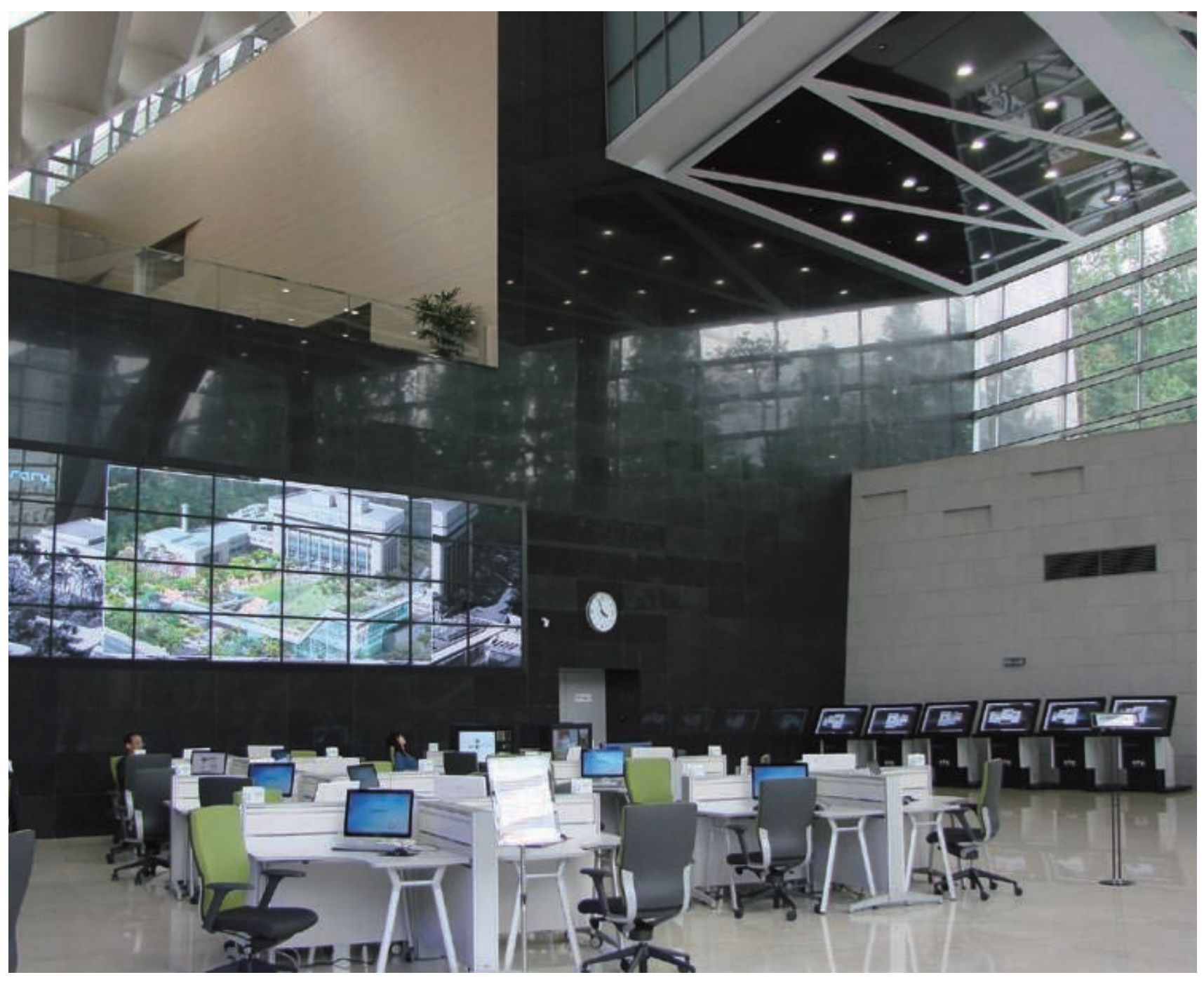

La Bibliothèque nationale de Corée :

à l'image de la bibliothèque scientifique de demain ? 


\section{Le numérique : une bibliothèque universelle pour la création scientifique?}

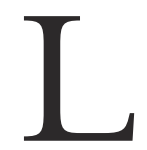

a réflexion qui suit trouve sa source dans l'expérience personnelle des auteurs et dans la pratique de leurs collègues français. Elle est propre aux sciences " exactes ", mais certains constats, évolutions ou perspectives concernent certainement aussi d'autres secteurs disciplinaires.

L'objet de ce propos est d'identifier le rôle et l'emploi de la documentation dans le processus d'élaboration cognitif qui mène le chercheur à la découverte, puis de cerner l'usage de la numérisation qui s'impose désormais dans son exploration des connaissances, ainsi que dans la diffusion de sa propre production.

L'analyse des incidences techniques de cette évolution nous conduit tout d'abord à décrire les nouvelles modalités de l'exploration bibliographique du chercheur et, en corollaire, à dessiner les nouveaux contours émergents de la bibliothèque universitaire.

Mais pour autant le support papier conserve une forte valeur ajoutée et, par ailleurs, les outils informatiques indispensables à la quête bibliographique requièrent un emploi expert pour que soit garantie la valeur de la connaissance collectée. Au-delà même, l'emploi inévitable de ces nouveaux instruments pour accéder au savoir a un impact sur la réflexion du chercheur, qui doit dorénavant se garder de l'encadrement insidieux qu'ils peuvent introduire dans le processus de construction de sa culture et de sa création scientifiques.

Libéré par l'accès à portée de main du savoir du monde, qui lui est offert par la numérisation, mais exposé à de nouvelles contraintes pour explorer fructueusement ce gigantesque vivier d'informations, le chercheur scientifique saura-t-il s'inventer une nouvelle démarche d'enrichissement qui soit propice à l'émergence de son étincelle créatrice?

\section{La documentation au centre de l'activité du chercheur}

Dans les domaines qui appartiennent aux sciences dites " exactes ", le chercheur construit sa réflexion en prolongement (ou en ricochet) de travaux déjà effectués dans la thématique étudiée, puis il en publie le fruit pour le confronter à l'avis critique de la communauté. Cet avis porte sur la pertinence (spéculative ou applicative) de cet apport, sur sa justesse technique et son caractère novateur. Puis, lorsque la connaissance que le chercheur s'est ainsi forgée constitue un corpus aux contours solides, sa mise en forme dans un ouvrage peut en relier les fondements, les développements et la portée.

Ainsi se tisse un rapport très serré entre le chercheur et la documentation scientifique, que celui-ci soit vu comme utilisateur ou comme producteur.

A priori, le processus cognitif de la réflexion que le chercheur en sciences dures établit à partir de sa documentation peut sembler différent selon la nature de sa recherche (fondamentale, appliquée ou technologique). C'est que le germe d'une découverte fondamentale naît de nouveaux " outils de pensée ", alors qu'une invention appliquée émerge d'un savoir pluridisciplinaire éclectique, et qu'une innovation technologique résulte souvent de la synergie de multiples techniques. Mais dans tous les cas, le processus de création que le chercheur met en œuvre utilise la documentation comme vecteur essentiel de son " bricolage intellectuel ", et il l'exploite selon les mêmes démarches.

Il s'attache, dans un premier temps, aux écrits qui sont à l'origine de sa thématique de recherche ; en approfondir les hypothèses est essentiel pour reconnaître la portée des résultats primordiaux dans son domaine, afin d'y adosser sa propre réflexion. Il élargit ensuite sa 
prospection aux publications récentes traitant de sa thématique (et de son voisinage) pour identifier les pistes déjà explorées, tirer enseignement de leurs résultats et impasses, donc pour cadrer sa propre contribution et l'originalité de celle-ci.

Mais paradoxalement, c'est l'exploration d'un autre réservoir de savoirs qui se révèle porteuse des plus belles promesses de créativité, même si elle est plus aventureuse, et incroyablement incertaine. Issus de la documentation relevant d'autres disciplines, parfois d'autres sciences, des schémas de pensée inexplorés dans le socle de connaissances de son propre domaine peuvent ouvrir, voire illuminer, le processus cognitif qui aboutit à la découverte du chercheur.

Ce dernier mode d'investigation a pu être incroyablement fécond, comme en atteste l'histoire des sciences, lorsqu'on parvient à adapter un principe découvert ailleurs à la résolution de son propre problème. D’Alembert, déjà, insistait sur cet équilibre entre réalité et abstraction, présenté comme le juste milieu à adopter entre observation et mathématisation.

A son tour, quand par la suite il se fait auteur, le chercheur peut avoir une ambition plus universelle que la seule parution de ses résultats dans les articles ou ouvrages concernant sa spécialité. Ayant lui-même pu faire l'expérience d'une interrogation socratique qui nettoie les neurones, il sait combien pourront être stimulantes et éclairantes les utilisations de son domaine par des scientifiques venus d'autres cieux. Le champ disciplinaire où publier est complètement ouvert au chercheur, pour peu qu'il soit porté à transmettre ailleurs son savoir.

\section{Le numérique dans la relation du chercheur au monde}

Cependant, depuis l'arrivée du numérique, différents facteurs ont progressivement provoqué la mutation des modalités mêmes de la pratique documentaire.

C'est que, progressivement, cette nouvelle réalité technologique a suscité la standardisation de codages compatibles multisupports, ainsi que la constitution de bases de données spécialisées qui mettent à disposition pratique les documents scientifiques dans leur version numérisée, et ce dès leur production.

S'y ajoute une nouvelle réalité économique. En effet, l'édition concentrée de ces documents par un nombre restreint de grands groupes internationaux, et leur publication en une langue unique, l'anglais, ont limité la dispersion des sites à explorer et facilité la constitution de vastes réservoirs de données.

Enfin, la compétition entre chercheurs s'exacerbe, illustrée par la redoutable injonction emblématique publish or perish (publier ou périr), parfois au-delà de toute raison. La nature même des travaux entrepris en sciences " exactes " (à savoir la production de connaissances) et la nécessité de faire reconnaître la paternité de ses résultats engendrent désormais l'édition numérisée continue d'un grand volume de documents scientifiques à l'échelle mondiale.

Suite à ces évolutions, le numérique est devenu rapidement le premier format de stockage des documents explorés par le chercheur-lecteur scientifique. Logiquement, la prospection de ces stocks a nécessité le développement de moteurs de recherche appuyant leurs algorithmes sur une indexation des contenus ; celle-ci remplace le catalogage effectué à partir de métadonnées d'autorité (tel celui de la BnF) par l'indexation de mots-clés ou de métadonnées enrichies, relevés dans les documents euxmêmes. Il faut souligner le caractère indispensable de l'aide ainsi apportée pour isoler les travaux effectués sur un sujet particulier, sur une démarche, une théorie, un domaine d'expérimentation...

Puis, par extension, l'utilisation de langages de balisage permet désormais d'indexer des formats intégrant des éléments multimedia variés : textes, images, vidéos, sons, séquences interactives, voire (avec iPython, par exemple) expressions mathématiques et exécutions de programmes.

Enfin, sont à l'étude des analyses de nature sémantique, capables de guider la recherche de schémas de raisonnements, de corrélations de concepts, de relations de dépendances entre théorie et propriété... On pourrait ainsi, par exemple, retrouver aisément les différentes 


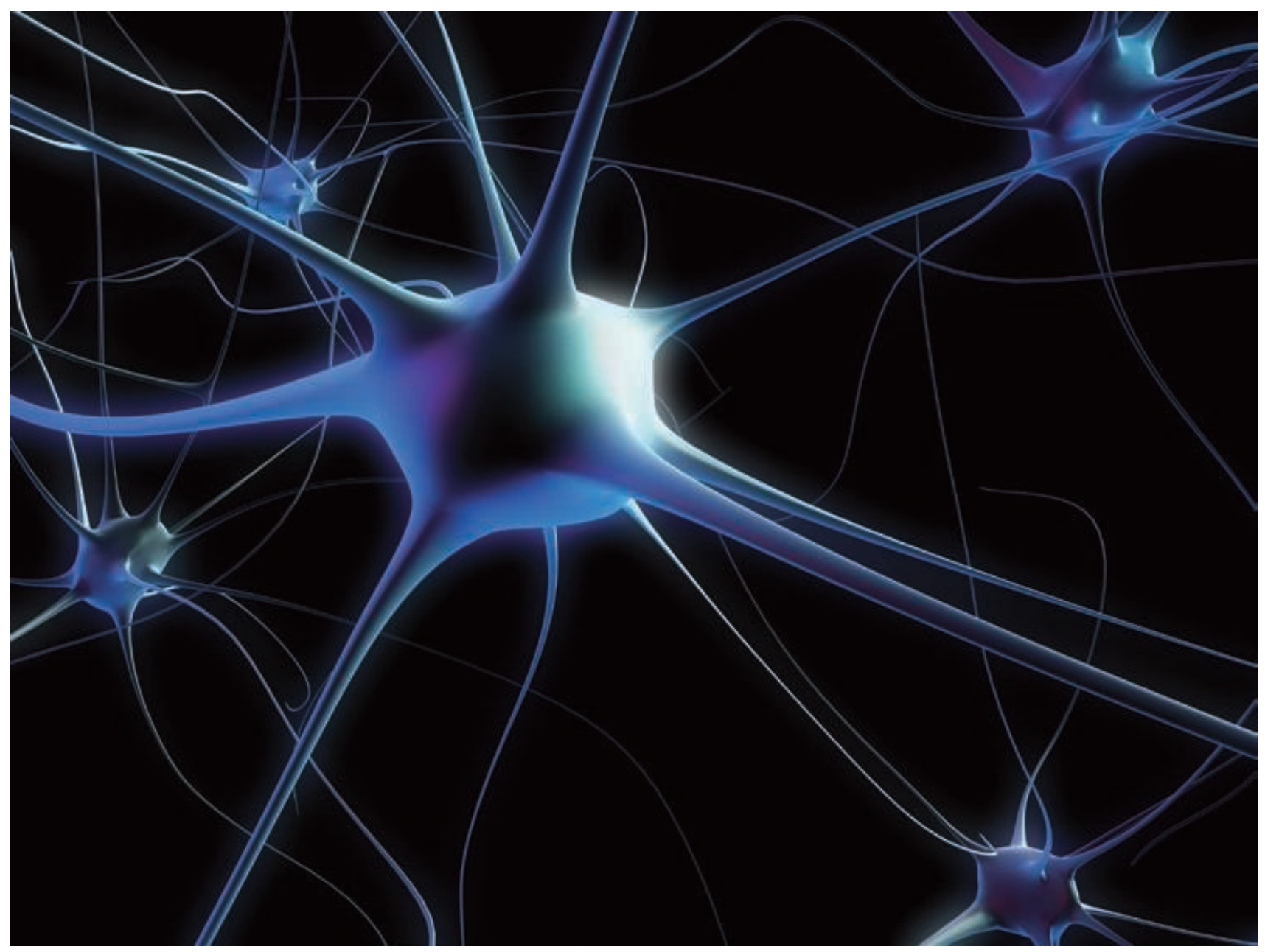

Neurone, cellule, par Eraxion (août 2009). Illustration de neurones avec leurs ramifications dendritiques, de serveurs informatiques connectés en réseau... ou du processus créatif du chercheur? 
versions d'un même article, et distinguer la version génératrice d'une idée féconde de celle qui en expose une utilisation pratique, et de celle qui est rédigée dans un but pédagogique.

Le chercheur scientifique effectue désormais son exploration bibliographique en exploitant de tels moteurs sur des bases de données d'éditeurs payantes (ScienceDirect d'Elsevier, SpringerLink de Springer Verlag, Web of Science de Thomson Reuters, etc.), celles de portails d'accès privés multiéditeurs (comme Inist du CNRS), ou de l'outil de recherche " universel " gratuit Google Scholar.

Dans son activité d'auteur aussi, le chercheur scientifique s'appuie sur le numérique, en utilisant de nombreux progiciels qui facilitent considérablement la rédaction d'articles scientifiques en version numérisée et qui en accompagnent la construction partagée, aussi bien avec les autres auteurs qu'avec l'éditeur. Un chercheur dispose maintenant gratuitement de logiciels de composition de documents (comme LaTeX pour l'écriture d'équations mathématiques), des outils de rédaction coopératifs (comme Sun, Git ou Google Docs), des applications pour l'échange de fichiers en ligne (comme Dropbox). Il rédige désormais ses articles et ouvrages en y intégrant les règles de style de l'éditeur : ils sont donc produits par l'auteur dans leur mise en page quasi définitive. La soumission au comité éditorial est automatisée, la parution accélérée, les coûts réduits.

Enfin, on a vu que la qualité professionnelle d'un chercheur est " évaluée " au vu du nombre de ses publications et, plus encore, du nombre de citations de ses publications par d'autres auteurs. Or ces références sont dénombrées à partir de l'analyse des bases de données documentaires. Le passage au numérique est ainsi devenu une transition qui s'impose au chercheur pour faire reconnaître la qualité de sa production : en l'affichant sur un site internet scientifique dans sa version numérisée, elle sera connue et référencée. Ce site peut être celui d'un éditeur, mais ce peut être aussi celui d'une université, d'un laboratoire de recherche, d'un établissement à la notoriété reconnue, en particulier par son comité éditorial ou la qualité de ses travaux.

\section{Quel impact sur le " vivre " du chercheur?}

Au-delà de l'évolution des modalités techniques, il devient aujourd'hui important de s'interroger sur l'impact que peut avoir l'accès à une documentation actualisée en permanence sur le processus cognitif du chercheur, qui le conduit à la création. On ne peut pas non plus éviter de considérer comme obsolète la notion classique de fonds documentaire et donc d'envisager une transformation conséquente des bibliothèques universitaires scientifiques.

Le chercheur scientifique adopte désormais une façon de construire sa réflexion qui s'appuie sur les nouvelles modalités d'interaction introduites par le numérique. Comment imaginer qu'il puisse mettre en œuvre sa problématique sur la seule base d'un corpus figé de connaissances alors que la recherche vit dans un bouillonnement incessant ? Sa démarche de création ne peut plus être linéaire, c'est-à-dire s'édifier sur un socle établi de savoirs. Il doit être en synchronisation constante avec l'émission de nouvelles idées, la parution des nouvelles avancées. Sa création intellectuelle émerge maintenant de la floraison d'informations qui naissent, se modifient, s'approfondissent ou deviennent caduques au contact des travaux du monde entier. Elle se stabilise au moment où en émerge une conclusion nouvelle, cohérente. Alors, à son tour, ce résultat s'intègre dans l'enrichissement scientifique global.

Une nouvelle relation s'établit ainsi entre le chercheur et sa documentation. Elle engendre un cadre pour sa création en rupture franche avec le passé. Son édification prend appui sur des bases dynamiques d'annotations, d'enregistrements audio et vidéo, de réalisations simulées, d'applications téléchargées, de conférences " podcastées ", le tout organisé par des index évolutifs, structuré par des arbres sémantiques. La bibliothèque du chercheur devient ainsi une arborescence en enrichissement permanent de données interconnectées ; elle est tissée avec sa propre production et virtualisée " dans le nuage " ; il y accède et y contribue en tous lieux et à tous moments, seul ou en partage.

En 1956, Alain Resnais réalise un court-métrage qui imagine l'évolution de la Bibliothèque nationale. Il y présage que celle-ci accueillera trois millions de volumes par siècle, que son bâtiment s'enfoncera de plus en plus dans le sol, et s'élèvera dans le ciel. On a dû en répertorier les ouvrages dans des catalogues volumineux à partir de mots-clés ; la salle des catalogues est devenue le cerveau de la $\mathrm{BN}$, elle gère $100 \mathrm{~km}$ de rayonnages ${ }^{1}$.

Dans le secteur scientifique, cette vision de la bibliothèque du futur est à l'opposé de l'évolution en gestation. Certains établissements supérieurs et laboratoires de recherche s'interrogent actuellement sur la pérennité des bibliothèques universitaires sous leur forme actuelle, au 


\section{La bibliothèque du chercheur devient une arborescence en enrichissement permanent de données interconnectées.}

moins dans la branche des sciences " exactes ". Le débat est intense. Les perspectives laissent prévoir une mutation en profondeur. La BU devient un espace aménagé pour la réflexion et le partage, où est offert l'accès aux versions numériques des revues prises en abonnement, ainsi qu'aux bases de données accessibles. La mise à disposition des documents sous forme papier se raréfie, et les services de conseil associés ne sont plus assurés que par la bibliothèque centrale, qui est loin.

Or cette mutation envisagée des bibliothèques scientifiques ne serait-elle pas déjà dépassée ? A l'image du téléphone qui, fixe, désigne un lieu et, mobile, est attaché à une personne, la bibliothèque du chercheur scientifique devient progressivement personnelle et mobile, détachée de toute localisation géographique. L'apprenti chercheur de demain exploitera une galaxie de connaissances en croissance infinie. Il la consommera et l'alimentera en usant des nouveaux modes de comportement que l'informatique a introduits. C'est que celle-ci a dépassé le stade du simple outil qui, de sa neutralité formelle (codage, traitement numérique), irrigue l'ensemble des sciences. Dans tous les secteurs d'activité, on observe qu'elle modifie profondément "la façon de penser le monde " en transformant les rapports de l'homme social avec l'espace, le temps, la communication, les rapports entre communautés.

Ce changement, à l'évidence, affecte en premier lieu le chercheur. Il travaille désormais dans un contexte très connecté qui lui impose des usages de partage et d'échange inédits : langages sociolectes, reformulation de la propriété intellectuelle, connectivité universelle et sans limite de flux, abolition des dépendances spatiales, désynchronisation des échanges, regroupements en réseaux spécialisés, volatiles et à coopération spontanée, agilité décisionnelle.

En sciences exactes, l'idée même d'une " bibliothèque universelle ", collection ordonnée de vérités suffisant à la déduction de toutes choses utiles, telle qu'envisagée par Leibniz, est devenue chimérique.

\section{Usage et précautions de la documentation numérique}

Cependant, malgré tous ses apports indiscutables, le numérique n'est pas encore parvenu à maturité, et il suscite chez le chercheur un nouveau comportement qui le confronte à de nouvelles questions, majeures.

D’une part, le numérique actuel présente des limites, appelées à être dépassées. Techniquement, les formats numériques multisupports qui codent désormais les textes (PDF et EPUB) permettent aussi de manipuler des documents enrichis de formulaires interactifs, de simulateurs numériques, de vidéos, etc. Cette possibilité devrait annoncer la floraison de ressources scientifiques didactiques nouvelles. Elle rend en effet possible de tester directement dans le texte les notions et les techniques qui y sont exposées, et de les assimiler par des exercices interactifs. Mais pour l'instant, l'édition scientifique n'en fait pas un véritable usage, ne serait-ce que parce l'intégration d'éléments multimédia dans un texte requiert encore des compétences informatiques particulières, en JavaScript, par exemple, et accroît donc le coût final du document. 
Pourtant, il s'agit sans doute là de la clef de l'évolution des ouvrages de référence pour les étudiants de licence, master ou doctorat, car se dessine déjà de leur part la demande, non seulement de nouvelles connaissances, mais aussi de nouvelles compétences dans la construction de leur documentation numérisée. Et ce sont eux, les chercheurs de demain.

A l'opposé subsiste encore puissamment la question de l'intérêt du support papier. Si le format numérique s'est indiscutablement imposé au chercheur-lecteur, ce n'est pas pour autant la seule voie d'exploration de l'actualité scientifique : la revue papier dont on balaie l'ensemble des articles, le magazine de synthèse disciplinaire, l'ouvrage thématique qu'on feuillette sont tous des déclencheurs potentiels de créativité. En outre, une fois identifiée la documentation numérique utile à ses travaux, le chercheur procède bien souvent à son analyse sur son édition papier ; le document imprimé facilite en effet le fléchage mental entre concepts, équations et résultats qui sont répartis dans le document. Il laisse aussi toute liberté pour les annotations sous des formes très personnelles, comme des croquis, des portions d'équations, des commentaires ébauchés, qui deviennent des tremplins à la poursuite de la réflexion. La conjugaison des deux supports engendre un nouveau régime de lecture chez les chercheurs scientifiques, qui se met en place depuis près de dix ans, et n'a probablement pas fini d'évoluer.

En tant qu'auteur, la question " papier ou numérique " ne se pose pas de la même façon pour le chercheur. Toutes les revues des sciences exactes ont une version numérique. Les conférences n'éditent plus guère d'actes, qui sont délivrés sur support numérique ou sur site internet. Reste le format de parution des ouvrages : papier exclusivement, papier et numérique (en version non reproductible), extraits numériques gratuits, voire intégralité gratuite en version numérique (et papier payant)... Toutes les options sont actuellement tentées pour concilier la diffusion scientifique et son modèle économique.

Sans nul doute, le papier, en tant que support des documents scientifiques, diminue très sensiblement en volume. Un équilibre à terme avec le numérique est vraisemblable, avec des différences qui se dessineront dans les usages ou dans les finalités. Mais ce dénouement est encore bien confus.

Enfin, le chercheur se pose inévitablement la question très inconfortable : quel crédit peut-on accorder aux moteurs de recherche des bases documentaires? Google Scholar s'impose progressivement comme outil de veille des nouvelles parutions. On peut y intégrer des motsclés générateurs d'alertes, le paramétrer pour qu'il notifie les nouvelles publications d'un chercheur référencé, ou pour qu'il signale les publications citant ce chercheur. Ces fonctionnalités et sa gratuité font son succès. Mais comme les autres moteurs, les premiers travaux qu'il affiche en résultat d'une recherche indexée ne sont pas nécessairement les plus significatifs scientifiquement, ni même les plus cités.

Le placement en première position d'un document ne présage donc rien de sa valeur scientifique supposée, et sa vérification à la source reste indispensable. En conséquence, dans son rôle de producteur de contenu, le chercheur-auteur doit prêter une attention toute particulière au titre et aux mots-clés qu'il associe à ses publications ; les choix les plus percutants ne sont généralement pas les plus appropriés au " moissonnage " numérique.

En outre, pour éviter les retours erronés du processus d'indexation, il devient impératif d'utiliser une nomenclature universelle pour les publications (le DOI-Digital Object Identifier se met en place), mais aussi pour les auteurs : les confusions entre chercheurs homonymes sont courantes!

La neutralité de la bibliographie collectée par les moteurs actuels est donc toute relative. Naturellement, l'évolution de leurs algorithmes, la mise en place de nouveaux standards de référencement pourront, peu à peu, remédier à ces défauts. Mais restera une interrogation fondamentale : l'emploi de ces techniques d'exploration pourrait bien, si l'on n'y prend garde, freiner autant qu'ouvrir la créativité du chercheur.

\section{Trouver un nouveau temps}

Un vertige peut saisir le chercheur d'aujourd'hui, à tenter de suivre, dans une course affolée, les nouveaux apports produits dans sa communauté scientifique tous les jours. Ce vertige peut s'accompagner d'angoisse quand il s'aperçoit que les outils qui lui servent à maîtriser ce flux incessant d'informations peuvent lui délivrer des résultats biaisés. Il doit maintenant trouver sa distance avec ce feu si intense. Il lui faut se réapproprier le temps d'une réflexion solitaire apaisée qui est indispensable à l'émergence d'une construction scientifique approfondie, et donc d'une véritable contribution au savoir humain.

\section{Jean-Pierre Elloy, Morgan Magnin}




\section{Notes}

1 - A. Resnais, réalisateur. Toute la mémoire du monde. Films de la Pléiade, 21 min. (1956)

2 - Voir M. Serres, Petite Poucette. Discours en séance solennelle à l'Académie française (2011)

\section{ORIENTATIONS BIBLIOGRAPHIQUES :}

G. Berry, Ch. Deshayes / Les Inversions mentales de l'informatique, in Journal de l'École de Paris du management, 2011

J.-P. Changeux, A. Connes | Matière à pensée, Odile Jacob, 1989

J. d'Alembert / Discours preliminaire de l'Encyclopédie, in L'Encyclopédie, ou Dictionnaire raisonné des sciences, des arts et des métiers, tome 1, 1751

R. Darnton / The new Age of the Book, in The New York Review, 1999. Analyse par R. Robert : L'édition électronique existe-t-elle?, in Esprit, 1999

J. Dhombres / Structures mathématiques et formes de pensée chez les ingénieurs, in Culture technique, 1984

A. W. K. Harzing, R. Van der Wal / Google Scholar as a new source for citation analysis?, in Ethics in Science and Environmental Politics, 2008

B. Latour, S. Woolgar / The construction of scientific facts, $2^{\mathrm{e}}$ édition, Princeton University Press, 1986

Cl. Lévi-Strauss / La Pensée sauvage, Plon, 1962

J. M. Noyes, K. J. Garland / Computer vs paper-based tasks: Are they equivalent?, in Ergonomics, septembre 2008

A. Resnais, réalisateur / Toute la mémoire du monde, Films de la Pléiade, 21 min., 1956

Ch. Robin / Les livres dans l'univers numérique, La documentation française, 2011

M. Serres / Petite Poucette, discours en séance solennelle à l'Académie française, 2011

B. Stiegler (sous la dir. de) / Le Design de nos existences à l'époque de l'innovation ascendante, Mille et une nuits, 2008

M. Tessier, B. Racine et al. / La Révolution du livre numérique, Odile Jacob, 2008 